\title{
The formation of free-floating brown dwarves and planetary-mass objects by photo-erosion of prestellar cores
}

\author{
A. P. Whitworth ${ }^{1}$ and H. Zinnecker ${ }^{2}$ \\ 1 School of Physics \& Astronomy, Cardiff University, 5 The Parade, Cardiff CF24 3YB, Wales, UK \\ e-mail: ant@astro.cf.ac.uk \\ 2 Astrophysikalisches Institut Potsdam, An der Sternwarte 16, 14482 Potsdam, Brandenburg, Germany \\ e-mail: hzinnecker@aip.de
}

Received 21 April 2004 / Accepted 22 July 2004

\begin{abstract}
We explore the possibility that, in the vicinity of an OB star, a prestellar core which would otherwise have formed an intermediate or low-mass star may form a free-floating brown dwarf or planetary-mass object, because the outer layers of the core are eroded by the ionizing radiation from the OB star before they can accrete onto the protostar at the centre of the core. The masses of objects formed in this way are given approximately by $\sim 0.010 M_{\odot}\left(a_{\mathrm{I}} / 0.3 \mathrm{~km} \mathrm{~s}^{-1}\right)^{6}\left(\dot{\mathcal{N}}_{\mathrm{Lyc}} / 10^{50} \mathrm{~s}^{-1}\right)^{-1 / 3}\left(n_{0} / 10^{3} \mathrm{~cm}^{-3}\right)^{-1 / 3}$, where $a_{\mathrm{I}}$ is the isothermal sound speed in the neutral gas of the core, $\dot{\mathcal{N}}_{\text {Lyc }}$ is the rate of emission of Lyman continuum photons from the OB star (or stars), and $n_{0}$ is the numberdensity of protons in the HII region surrounding the core. We conclude that the formation of low-mass objects by this mechanism should be quite routine, because the mechanism operates over a wide range of conditions $\left(10^{50} \mathrm{~s}^{-1} \lesssim \dot{\mathcal{N}}_{\mathrm{Lyc}} \lesssim 10^{52} \mathrm{~s}^{-1}\right.$, $10 \mathrm{~cm}^{-3} \lessgtr n_{0} \lesssim 10^{5} \mathrm{~cm}^{-3}, 0.2 \mathrm{~km} \mathrm{~s}^{-1} \lessgtr a_{\mathrm{I}} \lesssim 0.6 \mathrm{~km} \mathrm{~s}^{-1}$ ) and is very effective. However, it is also a rather wasteful way of forming low-mass objects, in the sense that it requires a relatively massive initial core to form a single low-mass object. The effectiveness of photo-erosion also implies that that any intermediate-mass protostars which have formed in the vicinity of a group of OB stars must already have been well on the way to formation before the OB stars switched on their ionizing radiation; otherwise these protostars would have been stripped down to extremely low mass.
\end{abstract}

Key words. stars: formation - stars: low-mass, brown dwarfs - ISM: HII regions

\section{Introduction}

The existence of brown dwarves was first proposed on theoretical grounds by Kumar (1963a,b) and Hayashi \& Nakano (1963), but more than three decades passed before observations were able to establish their existence unambiguously (Rebolo et al. 1995; Nakajima et al. 1995; Oppenheimer et al. 1995). Now they are routinely observed (e.g. McCaughrean et al. 1995; Luhman et al. 1998; Wilking et al. 1999; Luhman \& Rieke 1999; Lucas \& Roche 2000; Martín et al. 2000; Luhman et al. 2000; Béjar et al. 2001; Martín et al. 2001; Wilking et al. 2002; McCaughrean et al. 2002). It appears that a significant fraction of brown dwarves in clusters are not companions to more massive stars, but rather they are free-floating. Reipurth \& Clarke (2001) have suggested that this is because brown dwarves are ejected from nascent multiple systems before they can acquire sufficient mass to become Main Sequence stars, and this suggestion is supported by the numerical simulations of Bate et al. (2002), Delgado Donate et al. (2003) and Goodwin et al. (2004a,b). Here we explore an alternative possibility, namely that in the central regions of clusters like the Orion Nebula Cluster, isolated brown dwarves can form from pre-existing prestellar cores which are eroded by the ambient ionizing radiation field. Similar explanations for the formation of free-floating low-mass objects have been proposed by Zapatero Osorio et al. (2000) and Kroupa (2002), but they do not appear to have been explored in detail before now.

If a dense neutral core is immersed in an HII region, the ambient Lyman continuum radiation drives an ionization front into the core. The ionization front is normally preceded by a compression wave, and this may render the core gravitationally unstable. At the same time, the flow of ionized gas off the core reduces its mass and injects material and kinetic energy into the surrounding HII region. This situation was analyzed by Dyson (1968; see also Kahn 1969) who argued that it could explain the broadening and splitting of emission lines from the Orion Nebula. Dyson also considered the circumstances under which such a core might become gravitationally unstable.

Bertoldi (1989) and Bertoldi \& McKee (1990) have developed semi-analytic models for larger cometary globules created by the interaction of ionizing radiation with a preexisting cloud on the edge of an HII region. They use these semi-analytic models to evaluate the acceleration of the globule (due to the rocket effect; cf. Kahn 1954; Oort \& Spitzer 1955) and its gravitational stability. Their results agree broadly 
with the numerical simulations of Sandford et al. (1982) and Lefloch \& Lazareff (1994). More recently Kessel-Deynet \& Burkert (2002) have modelled the radiation driven implosion of a pre-existing large cloud having internal structure. They use Smoothed Particle Hydrodynamics to handle the threedimensional, self-gravitating gas dynamics, and find that smallscale internal structure delays, and may even inhibit, gravitational collapse.

However, in this paper we are concerned - like Dyson and Kahn - with small-scale neutral cores immersed well within an HII region, in the vicinity of the exciting stars, rather than large cometary globules at the edge of an HII region. In particular, we wish to evaluate the competition between erosion of material from the outer layers of such a core (due to the ionization front which eats into the core), and the formation and growth of a central protostar (triggered by the compression wave driven into the core ahead of the ionization front).

\section{Overview of model}

In order to keep free parameters to a minimum in this exploratory analysis, we make a number of simplifying assumptions. We assume that there exists a prestellar core in equilibrium, and that a nearby massive star (or group thereof) then switches on its ionizing radiation instantaneously, and ionizes the surroundings of the prestellar core, on a time-scale much shorter than the sound-crossing time for the core (we justify this assumption later). The resulting increase in external pressure triggers the collapse of the core, from the outside in, and at the same time an ionization front starts to eat into the core, thereby eroding it from the outside, as it contracts. The final stellar mass is therefore determined by a competition between the rate of accretion onto the central protostar, and the rate of erosion at the boundary. Erosion ceases to be effective when the ionization front has eaten so far into the collapsing core that the newly ionized gas flowing off the ionization front is despite its outward kinetic energy - still gravitational bound to the protostar.

The evolution consists of three phases, starting from the instant $t=0$ when the surroundings of the prestellar core first become ionized. At this juncture a compression wave is driven into the core setting up a uniform, subsonic inward velocity field. When this wave impinges on the centre at time $t_{1}$, the first phase terminates and a central protostar is formed, which then grows steadily by accretion. At the same time an expansion wave is reflected and propagates outwards leaving an approximately freefall velocity field in its wake. At time $t_{2}$ this outward propagating expansion wave encounters the inward propagating ionization front, and the second phase terminates. Thereafter the ionization front is eroding material which is falling ever faster inwards. Eventually, at time $t_{3}$, the ionization front encounters material which is falling inwards so fast that it cannot be unbound by the ionization front, and the third phase terminates. The final stellar mass is the mass interior to the ionization front at time $t_{3}$.

In Sect. 3 we derive an expression for the diffuse ionizing radiation field in an HII region, and in Sect. 4 we formulate the speed with which the ionization front eats into the core.
In Sect. 5, we describe the initial prestellar core and formulate the time $t_{1}$ it takes for the compression wave to propagate to its centre. In Sect. 6 we describe the modified density and velocity field set up in the inner core by the outward propagating expansion wave, and formulate the time $t_{2}$ at which the outward propagating expansion wave meets the inward propagating ionization front. In Sect. 7 we formulate the time $t_{3}$ at which the ionization front has eaten so far into the modified density field inside the expansion wave that it has encountered material which is infalling too fast to be unbound by the act of ionization. The material interior to the ionization front at time $t_{3}$ is presumed to constitute the final star. We can therefore obtain an expression for its mass.

\section{The diffuse ionizing flux}

We shall assume that the HII region which engulfs the preexisting prestellar core is excited by a compact cluster of OB stars which emits Lyman continuum (i.e. hydrogen ionizing) photons at a constant rate $\dot{\mathcal{N}}_{\text {Lyc }}$. We shall also assume that most of the surrounding space is occupied by a uniform gas in which the density of hydrogen nuclei in all forms is $n_{0}$. The radius of the initial HII region is therefore

$R_{\mathrm{HII}}=\left(\frac{3 \dot{\mathcal{N}}_{\mathrm{Lyc}}}{4 \pi \alpha_{*} n_{0}^{2}}\right)^{1 / 3}$,

where $\alpha_{*} \simeq 2 \times 10^{-13} \mathrm{~cm}^{3} \mathrm{~s}^{-1}$ is the recombination coefficient into excited states only; in other words, we are using the OnThe-Spot Approximation.

If we neglect the shadows cast by other prestellar cores and attenuation by dust inside the HII region, the radial - or direct number-flux of Lyman continuum photons $\dot{N}_{\text {Lyc }}^{\text {radial }}$ (i.e. the number of ionizing photons crossing unit area in unit time) varies with distance $R$ from the OB cluster as

$\dot{N}_{\text {Lyc }}^{\text {radial }}=\frac{\dot{\mathcal{N}}_{\mathrm{Lyc}}}{4 \pi R^{2}}-\frac{\alpha_{*} n_{0}^{2} R}{3}$

where the first term on the righthand side represents geometric dilution and the second term represents the absorptions which maintain ionization against recombination. Hence the mean value of $\dot{N}_{\text {Lyc }}^{\text {radial }}$, averaged over the volume of the HII region, is

$\left\langle\dot{N}_{\mathrm{Lyc}}^{\text {radial }}\right\rangle \simeq \frac{3}{4}\left(\frac{3 \dot{\mathcal{N}}_{\mathrm{Lyc}} \alpha_{*}^{2} n_{0}^{4}}{4 \pi}\right)^{1 / 3}$.

The volume number-emissivity for the diffuse component of the Lyman continuum radiation field (i.e. the number of ionizing photons emitted from unit volume in unit time into unit solid angle, due to recombinations straight into the ground state) is

$\dot{n}_{\text {Lyc }}^{\text {diffuse }}=\frac{\alpha_{1} n_{0}^{2}}{4 \pi}$,

where $\alpha_{1} \simeq 10^{-13} \mathrm{~cm}^{3} \mathrm{~s}^{-1}$ is the recombination coefficient into the ground state only. The mean diffuse flux is therefore of order

$\left\langle\dot{N}_{\text {Lyc }}^{\text {diffuse }}\right\rangle \sim \pi \dot{n}_{\text {Lyc }}^{\text {diffuse }} R_{\mathrm{HII}} \simeq \frac{\alpha_{1}}{4 \alpha_{*}}\left(\frac{3 \dot{\mathcal{N}}_{\mathrm{Lyc}} \alpha_{*}^{2} n_{0}^{4}}{4 \pi}\right)^{1 / 3}$. 
Comparing Eqs. (3) and (5), we see that the mean diffuse flux is smaller than the mean radial flux by a factor $\sim \alpha_{1} / 3 \alpha_{*} \sim 1 / 6$. This is to some extent compensated by the fact that a spherical core of radius $r$ presents a larger effective cross-section to the diffuse flux $\left(4 \pi r^{2}\right)$ than to the radial flux $\left(\pi r^{2}\right)$, i.e. the net dose of Lyman continuum radiation received by a spherical core divides roughly in the ratio $60 \%$ radial and $40 \%$ diffuse. In order to proceed with a spherically symmetric analysis, we shall assume that the flux of Lyman continuum radiation incident on a core is constant, isotropic, and equal to $2\left\langle\dot{N}_{\text {Lyc }}^{\text {diffuse }}\right\rangle$. However, we should be mindful that this is a crude representation of the Lyman continuum radiation field incident on a real core in a real HII region. We will check retrospectively that the cores we invoke are much smaller than the HII region in which they are imersed (see Sect. 8).

\section{The photo-erosion rate}

We denote the instantaneous radius of the neutral core by $r_{\mathrm{IF}}$, and the density of hydrogen nuclei in all forms in the neutral core by $n_{\mathrm{I}}(r), r \leq r_{\mathrm{IF}}$, where $r$ is distance measured from the centre of the core. We assume that the ionization front eats into the core at speed $\left[-\dot{r}_{\mathrm{IF}}\right]<a_{\mathrm{I}}$, where $a_{\mathrm{I}}$ is the isothermal soundspeed in the neutral gas. Consequently the ionization front is not preceded by a strong shock front, just a weak compression wave. This is a reasonable assumption, except near the beginning, when the ionization front advances very rapidly into the core. We can only allow for this by performing numerical simulations. However, this initial phase of rapid erosion does not last long, and will not greatly affect the overall phenomenology of core erosion or the final protostellar mass. It will simply shorten the time interval, $t_{1}$, before the protostar forms. Thus the expressions for the final protostellar mass which we derive later (Eqs. (40) and (41)) should be taken as indicative rather than precise.

Conservation of mass across the ionization front requires

$n_{\mathrm{I}}\left(r_{\mathrm{IF}}\right)\left[-\dot{r}_{\mathrm{IF}}\right]=n_{\mathrm{II}}\left(r_{\mathrm{IF}}\right)\left[v_{\mathrm{II}}\left(r_{\mathrm{IF}}\right)-\dot{r}_{\mathrm{IF}}\right]$,

where $n_{\mathrm{II}}\left(r_{\mathrm{IF}}\right)$ and $v_{\mathrm{II}}\left(r_{\mathrm{IF}}\right)$ are - respectively - the density of hydrogen nuclei in all forms and the radial speed of the ionized gas, just outside the neutral core.

To proceed, we assume that the ionized gas flows away from the ionization front at constant speed

$\left[v_{\mathrm{II}}(r)-\dot{r}_{\mathrm{IF}}\right]=\left[v_{\mathrm{II}}\left(r_{\mathrm{IF}}\right)-\dot{r}_{\mathrm{IF}}\right]=a_{\mathrm{II}}$,

where $a_{\mathrm{II}}$ is the isothermal sound-speed in the ionized gas. (In reality (Kahn 1969) the flow of ionized gas downstream from the ionization front accelerates away from the core due to the pressure gradient, but this only changes the result we derive below by a numerical factor of order unity.) Hence the density in the ionized gas falls off approximately as $r^{-2}$,

$n_{\mathrm{II}}(r)=n_{\mathrm{II}}\left(r_{\mathrm{IF}}\right)\left(\frac{r}{r_{\mathrm{IF}}}\right)^{-2}$,

until it approaches the background density $n_{0}$ at radius $r_{\infty} \gg$ $r_{\mathrm{IF}}$. Also, substituting from Eq. (7) into Eq. (6) gives

$n_{\mathrm{I}}\left(r_{\mathrm{IF}}\right)\left[-\dot{r}_{\mathrm{IF}}\right] \simeq n_{\mathrm{II}}\left(r_{\mathrm{IF}}\right) a_{\mathrm{II}}$.
Almost all the incident flux of Lyman continuum photons is used up balancing recombinations in the outflowing ionized gas, so we can put

$$
\begin{aligned}
& 2\left\langle\dot{N}_{\mathrm{Lyc}}^{\mathrm{diffuse}}\right\rangle \simeq \int_{r=r_{\mathrm{IF}}}^{r=r_{\infty}} \alpha_{*} n_{\mathrm{II}}^{2}(r) \mathrm{d} r \simeq \frac{\alpha_{*} n_{\mathrm{II}}^{2}\left(r_{\mathrm{IF}}\right) r_{\mathrm{IF}}}{3} ; \\
& n_{\mathrm{II}}\left(r_{\mathrm{IF}}\right) \simeq\left(\frac{6\left\langle\dot{N}_{\mathrm{Lyc}}^{\mathrm{diffue}}\right\rangle}{\alpha_{*} r_{\mathrm{IF}}}\right)^{1 / 2} ; \\
& \dot{r}_{\mathrm{IF}} \simeq-\frac{n_{\mathrm{II}}\left(r_{\mathrm{IF}}\right) a_{\mathrm{II}}}{n_{\mathrm{I}}\left(r_{\mathrm{IF}}\right)} \simeq-\left(\frac{6\left\langle\dot{N}_{\mathrm{Lyc}}^{\mathrm{diffuse}}\right\rangle}{\alpha_{*} r_{\mathrm{IF}}}\right)^{1 / 2} \frac{a_{\mathrm{II}}}{n_{\mathrm{I}}\left(r_{\mathrm{IF}}\right)}
\end{aligned}
$$

In obtaining the final expression in Eq. (10), we have assumed that $r_{\infty} \gg r_{\mathrm{IF}}$. Again this is a reasonable assumption except near the beginning.

\section{The first phase $\left(0<t<t_{1}\right)$}

\subsection{The initial core}

For the purpose of this exploratory calculation, we assume that the pre-existing prestellar core is a singular isothermal sphere. Generalizing the treatment to a non-singular (i.e. Bonnor-Ebert) isothermal sphere should not change the result greatly, but would necessitate a detailed numerical formulation of the problem; it would tend to reduce the mass of the final protostar, and hence assist in producing low-mass final objects.

In a singular isothermal sphere, the density of hydrogen nuclei in all forms, $n_{\mathrm{I}}(r)$ and the mass interior to radius $r, M(r)$, are given by

$$
\begin{aligned}
n_{\mathrm{I}}(r) & =\frac{a_{\mathrm{I}}^{2}}{2 \pi G m r^{2}}, \\
M(r) & =\frac{2 a_{\mathrm{I}}^{2} r}{G},
\end{aligned}
$$

where $m=m_{\mathrm{p}} / X=2.4 \times 10^{-24} \mathrm{~g}$ is the mass associated with one hydrogen nucleus when account is taken of other elements (in particular helium), $m_{\mathrm{p}}$ is the mass of a proton, and $X$ is the fractional abundance of hydrogen by mass. If the boundary density is $n_{0}$, the outer radius and total mass are initially

$$
\begin{aligned}
r_{0} & =\frac{a_{\mathrm{I}}}{\left(2 \pi G n_{0} m\right)^{1 / 2}}, \\
M_{0} & =\left(\frac{2}{\pi G^{3} n_{0} m}\right)^{1 / 2} a_{\mathrm{I}}^{3} .
\end{aligned}
$$

\subsection{The inward propagating compression wave}

When this core is first overrun by the HII region, at time $t=0$, the resulting increase in the external pressure drives a compression wave into the core, thereby triggering its collapse. We assume that the compression wave travels ahead of the ionization front. It leaves in its wake a subsonic - and approximately uniform - inward velocity field. The compression wave reaches the centre at time

$t_{1}=\frac{r_{0}}{a_{\mathrm{I}}}=\frac{1}{\left(2 \pi G n_{0} m\right)^{1 / 2}}$. 
i.e. after one sound-crossing time. We can now justify the assumption that the time-scale on which the HII region overruns the core is much shorter than a sound-crossing time. The timescale for the establishment of the Initial Strømgren Sphere is of order the recombination time-scale, viz.

$t_{\text {recombination }} \sim\left(\alpha_{*} n_{0}\right)^{-1}$,

so the condition becomes $t_{\text {recombination }} \ll t_{1}$, or

$n_{0} \gg \frac{2 \pi G m}{\alpha_{*}^{2}} \sim 3 \times 10^{-5} \mathrm{~cm}^{-3}$.

This is easily satisfied since typically $n_{0} \gtrsim 1 \mathrm{~cm}^{-3}$.

\section{The second phase $\left(t_{1}<t<t_{2}\right)$}

\subsection{Formation of the central protostar}

At $t=t_{1}$ a central protostar forms and subsequently grows in mass at a constant rate

$\dot{M}_{*} \simeq \frac{a_{\mathrm{I}}^{3}}{G}$

We note that this result is true for the collapse of a singular isothermal sphere irrespective of whether its collapse is triggered - as here - from outside-in by the inward propagation of a mild compression wave (see Whitworth \& Summers 1985), or - as in the Shu (1977) model - from inside-out by a central perturbation.

\subsection{The expansion wave}

At the same time that the compression wave converges on the centre, an expansion wave is launched outwards at speed $a_{\mathrm{I}}$, relative to the gas. If we neglect the small inward velocity already acquired by the gas due to the inward propagating compression wave, the radius of the expansion wave is given by

$r_{\mathrm{EW}} \simeq a_{\mathrm{I}}\left(t-t_{1}\right) \simeq a_{\mathrm{I}} t-r_{0}$.

We now introduce dimensionless variables

$\xi=\frac{r}{r_{0}}$

$\tau=\frac{t}{t_{1}}$

in terms of which the radius of the expansion wave is given by

$\xi_{\mathrm{EW}}=\tau-1, \quad \tau>1$.

\subsection{The core interior to the expansion wave}

Interior to the expansion wave, material flows inwards, to feed the constant accretion rate onto the central protostar, and quickly approaches a freefall-like profile. Therefore the density and velocity can be approximated by

$$
\left.\begin{array}{l}
n_{\mathrm{I}}^{\prime}(r)=(3 / 8 \pi G m) a_{\mathrm{I}}^{2} r_{\mathrm{EW}}^{-1 / 2} r^{-3 / 2}, \\
v_{\mathrm{I}}^{\prime}(r)=-(2 / 3) a_{\mathrm{I}} r_{\mathrm{EW}}^{1 / 2} r^{-1 / 2},
\end{array}\right\} t>t_{1}, r<r_{\mathrm{EW}} .
$$

There is a degree of freedom in specifying the coefficients of $n_{\mathrm{I}}^{\prime}(r)$ and $v_{\mathrm{I}}^{\prime}(r)$. We have chosen them so that (i) they are consistent with the constant accretion rate (see Eq. (18)); and (ii) the mass of the central protostar and the mass in the accretion flow interior to the expansion wave are equal. Formally the velocity changes discontinuously across the expansion wave, from zero outside to $-2 a_{\mathrm{I}} / 3$ inside; in reality the change will be much smaller since the inward propagating compression wave during the first phase will have set up an inward velocity of comparable magnitude (e.g. Whitworth \& Summers 1985).

\subsection{The ionization front exterior to the expansion wave}

Substituting for $n_{\mathrm{I}}\left(r_{\mathrm{IF}}\right)$ in Eq. (12) from Eq. (13), we obtain

$\frac{\mathrm{d} r_{\mathrm{IF}}}{\mathrm{d} t}=-\left(\frac{6\left\langle\dot{N}_{\mathrm{Lyc}}^{\text {diffuse }}\right\rangle}{\alpha_{*} r_{\mathrm{IF}}}\right)^{1 / 2} \frac{2 \pi G m a_{\mathrm{II}} r_{\mathrm{IF}}^{2}}{a_{\mathrm{I}}^{2}}$.

If we now introduce a third dimensionless variable

$\begin{aligned} \beta & =\left(\frac{6\left\langle\dot{N}_{\mathrm{Lyc}}^{\mathrm{diffuse}}\right\rangle r_{0}^{3}}{\alpha_{*}}\right)^{1 / 2} \frac{\pi G m a_{\mathrm{II}}}{a_{\mathrm{I}}^{3}} \\ & =\left(\frac{\pi 3^{8} \alpha_{1}^{6} G^{3} m^{3} \dot{\mathcal{N}}_{\mathrm{Lyc}}^{2}}{2^{7} \alpha_{*}^{8} a_{\mathrm{I}}^{6} n_{0}}\right)^{1 / 12} \frac{a_{\mathrm{II}}}{2 a_{\mathrm{I}}} .\end{aligned}$

Equation (24) reduces to

$\frac{\mathrm{d} \xi_{\mathrm{IF}}}{\mathrm{d} \tau}=-2 \beta \xi_{\mathrm{IF}}^{3 / 2}$

with solution

$\xi_{\mathrm{IF}}=[1+\beta \tau]^{-2}$.

To fix the constant of integration, we have used the initial condition, viz.: at $t=0, r_{\mathrm{IF}}=\xi_{\mathrm{IF}} r_{0}=r_{0}$; and hence at $\tau=0$, $\xi_{\mathrm{IF}}=1$.

\subsection{The ionization front meets the expansion wave}

The ionization front meets the expansion wave at time $t_{2}$ when $r_{\mathrm{IF}}=r_{\mathrm{EW}}$, or in terms of the dimensionless variables, at $\tau_{2}$ when $\xi_{\mathrm{IF}}=\xi_{\mathrm{EW}}=\xi_{2}$. Equating Eqs. (27) and (22), we obtain

$\left(1+\beta \tau_{2}\right)^{-2}=\tau_{2}-1$.

With typical values $\dot{\mathcal{N}}_{\text {Lyc }}=10^{50} \mathrm{~s}^{-1}$ and $n_{0}=10^{4} \mathrm{~cm}^{-3}$, we have $\left\langle\dot{N}_{\mathrm{Lyc}}^{\text {diffuse }}\right\rangle \simeq 3 \times 10^{11} \mathrm{~cm}^{-2} \mathrm{~s}^{-1}$. Putting $a_{\mathrm{I}}=0.3 \mathrm{~km} \mathrm{~s}^{-1}$ and $a_{\mathrm{II}}=10 \mathrm{~km} \mathrm{~s}^{-1}$, we obtain $\beta \simeq 25$. Hence, if we put

$\xi_{2}=\tau_{2}-1$,

it follows that $\xi_{2} \ll 1$, and Eq. (28) becomes

$\left[(1+\beta)+\beta \xi_{2}\right]^{2} \xi_{2}=1$,

with approximate solution

$\xi_{2} \simeq(1+\beta)^{-2} \sim 0.002$.

This is the dimensionless radius of the inward propagating ionization front when it meets the outward propagating expansion wave. 


\section{The third phase $\left(t_{2}<t<t_{3}\right)$}

\subsection{The ionization front interior to the expansion wave}

Once the inward propagating ionization front passes the outward propagating expansion wave, the ionization front encounters infalling gas with density and velocity given by Eq. (23). Equation (9) therefore has to be adjusted to account for the inward motion of the neutral gas:

$n_{\mathrm{I}}^{\prime}\left(r_{\mathrm{IF}}\right)\left[-\dot{r}_{\mathrm{IF}}+v_{\mathrm{I}}^{\prime}\left(r_{\mathrm{IF}}\right)\right] \simeq n_{\mathrm{II}}\left(r_{\mathrm{IF}}\right) a_{\mathrm{II}}$.

Substituting for $n_{\mathrm{I}}^{\prime}\left(r_{\mathrm{IF}}\right)$ and $v_{\mathrm{I}}^{\prime}\left(r_{\mathrm{IF}}\right)$ from Eq. (23) and for $n_{\mathrm{II}}\left(r_{\mathrm{IF}}\right)$ from Eq. (11), we obtain

$\dot{\xi}_{\mathrm{IF}}=-\frac{2(\tau-1)^{1 / 2}}{3}\left[\xi_{\mathrm{IF}}^{-1 / 2}+4 \beta \xi_{\mathrm{IF}}\right]$.

The solution is

$(\tau-1)^{3 / 2}=\xi_{2}^{3 / 2}+\frac{3}{8 \beta} \ln \left[\frac{1+4 \beta \xi_{2}^{3 / 2}}{1+4 \beta \xi_{\mathrm{IF}}^{3 / 2}}\right]$,

where we have fixed the constant of integration by requiring that $r_{\mathrm{IF}}=r_{\mathrm{EW}}=r_{2}$ at $t_{2}$; or equivalently $\xi_{\mathrm{IF}}=\xi_{\mathrm{EW}}=\xi_{2}=\tau_{2}-1$ at $\tau_{2}$.

To follow the ionization front into the third phase, we pick ever smaller values of $\xi_{\mathrm{IF}}\left(<\xi_{2}\right)$ and calculate $\tau$ from Eq. (34). The radial velocity of the ionization front is then given by $\dot{r}_{\mathrm{IF}}=$ $a_{\mathrm{I}} \dot{\xi}_{\mathrm{FF}}$, where $\dot{\xi}_{\mathrm{IF}}$ can be calculated from Eq. (33). The specific kinetic energy of the newly ionized gas, relative to the central protostar is therefore

$\epsilon_{\mathrm{KIN}}=\frac{1}{2}\left[\dot{r}_{\mathrm{IF}}+a_{\mathrm{II}}\right]^{2}=\frac{a_{\mathrm{I}}^{2}}{2}\left[\dot{\xi}_{\mathrm{IF}}+\frac{a_{\mathrm{II}}}{a_{\mathrm{I}}}\right]^{2}$.

Taking into account the mass of the protostar, $M_{*}=$ $G^{-1} a_{1}^{2} r_{0}(\tau-1)$, plus the mass in the accretion flow interior to the ionization front, $M_{\mathrm{I}}=G^{-1} a_{\mathrm{I}}^{2} r_{0}(\tau-1)^{-1 / 2} \xi_{\mathrm{IF}}^{3 / 2}$, the specific binding energy of this material is

$\epsilon_{\mathrm{BIND}}=\frac{G\left(M_{*}+M_{\mathrm{I}}\right)}{r_{\mathrm{IF}}}=a_{\mathrm{I}}^{2}\left\{\frac{(\tau-1)}{\xi_{\mathrm{IF}}}+\frac{\xi_{\mathrm{IF}}^{1 / 2}}{(\tau-1)^{1 / 2}}\right\}$.

We shall assume that ionization ceases to erode the core once the specific kinetic energy, $\epsilon_{\mathrm{KIN}}$ (Eq. (35)), is less than the specific binding energy, $\epsilon_{\mathrm{BIND}}$ (Eq. (36)). The mass $M_{3}=M_{*}+M_{\mathrm{I}}$ interior to the ionization front at this stage is the final mass of the protostar. This occurs at time $t_{3}=\tau_{3} t_{1}$, where

$\frac{1}{2}\left[\dot{\xi}_{\mathrm{IF}}\left(\tau_{3}\right)+\frac{a_{\mathrm{II}}}{a_{\mathrm{I}}}\right]^{2}=\frac{\left(\tau_{3}-1\right)}{\xi_{3}}+\frac{\xi_{3}^{1 / 2}}{\left(\tau_{3}-1\right)^{1 / 2}} ;$

and the final mass of the protostar is then given by

$\frac{M_{3}}{M_{0}}=\frac{1}{2}\left[\left(\tau_{3}-1\right)+\left(\tau_{3}-1\right)^{-1 / 2} \xi_{3}^{3 / 2}\right]$.

\section{Results}

The procedure for obtaining the final protostellar mass $M_{3}$ is therefore as follows. (i) Choose $\left(\dot{\mathcal{N}}_{\mathrm{Lyc}}, a_{\mathrm{I}}, n_{0}\right)$ and calculate $\beta$ from Eq. (25). (ii) Solve Eq. (30) for $\xi_{2}$. (iii) Solve Eq. (34) for $\xi_{\mathrm{IF}}(\tau),\left(\tau>1+\xi_{2}\right)$. (iv) Identify $\tau_{3}$ satisfying Eq. (37). (v) Compute $M_{3}$ according to Eq. (38).

Figure 1 shows, for three representative values of $\dot{\mathcal{N}}_{\mathrm{Lyc}}$ (i.e. (a) $10^{50} \mathrm{~s}^{-1}$; (b) $10^{51} \mathrm{~s}^{-1}$; and (c) $10^{52} \mathrm{~s}^{-1}$ ) loci of contant $M_{3}=0.001 M_{\odot}, 0.012 M_{\odot}$, and $0.078 M_{\odot}$ on the $\left(a_{\mathrm{I}}, \log _{10}\left[n_{0}\right]\right)$-plane. These values of $M_{3}$ are chosen because they represent, respectively, one Jupiter mass, the deuteriumburning limit, and the hydrogen-burning limit. Notionally objects in the mass range $0.012 M_{\odot} \lesssim M_{3} \lesssim 0.078 M_{\odot}$ are referred to as brown dwarves, whilst those in the mass range $M_{3} \lesssim 0.012 M_{\odot}$ are referred to as planetary-mass objects. We see that for a broad range of values of $\dot{\mathcal{N}}_{\mathrm{Lyc}}, n_{0}$ and $a_{\mathrm{I}}$, there exists the possibility to form free-floating brown dwarves and planetary-mass objects, provided suitable pre-existing cores are available to be photo-eroded.

In estimating the radiation field incident on a core, we have assumed that the volume occupied by the core is much smaller than the volume of the HII region around it (see Sect. 3). It is therefore necessary to check that this is the case. If we require the ratio between the initial volume of a core and the volume of the whole HII region to be less than some fraction $\eta$, then substituting from Eqs. (1) and (15) we obtain the condition

$n_{0}<n_{\max }(\eta)=\frac{\pi}{2}\left(\frac{3 \dot{\mathcal{N}}_{\mathrm{Lyc}} \eta}{\alpha_{*}}\right)^{2}\left(\frac{G m}{a_{\mathrm{I}}^{2}}\right)^{3}$.

$n_{\max }(\eta)$ is plotted in Figure 1 for $\eta=0.001$ (dashed curve) and $\eta=0.01$ (dash-dot curve). Evidently this is a significant constraint for $\dot{\mathcal{N}}_{\mathrm{Lyc}} \sim 10^{50} \mathrm{~s}^{-1}$, but for higher values of $\dot{\mathcal{N}}_{\mathrm{Lyc}}$ it is less important.

Since the protostar does not grow hugely during the Third Phase, we can approximate the results by adopting the mass at the end of the Second Phase. If in addition we assume $\beta \gg 1$, so that from Eq. (31) $\xi_{2} \simeq \beta^{-2}$, we can write

$$
\begin{aligned}
M_{3} & \simeq f \dot{M}_{*} t_{1} \xi_{2} \\
& \simeq \frac{f 2^{3} \alpha_{*} a_{\mathrm{I}}^{6}}{3 \alpha_{1} a_{\mathrm{II}}^{2} G^{2} m}\left(\frac{\alpha_{*}}{6 \pi^{2} \dot{\mathcal{N}}_{\mathrm{Lyc}} n_{0}}\right)^{1 / 3} \\
& \simeq 0.01 M_{\odot}\left(\frac{a_{\mathrm{I}}}{0.3 \mathrm{~km} \mathrm{~s}^{-1}}\right)^{6}\left(\frac{\dot{\mathcal{N}}_{\mathrm{Lyc}}}{10^{50} \mathrm{~s}^{-1}}\right)^{-1 / 3}\left(\frac{n_{0}}{10^{3} \mathrm{~cm}^{-3}}\right)^{-1 / 3}
\end{aligned}
$$

Here $f \simeq 1.7$ is a factor to account for the additional matter which accretes onto the protostar after the ionization fron meets the expansion wave. We can understand this factor as follows. In a collapsing singular isothermal sphere, the infalling mass interior to the expansion wave is equal to the mass already in the central protostar. Thus, in the problem considered here, when the ionization front meets the expansion wave at $t=t_{3}$, the infalling mass interior to the ionization front equals the mass already in the protostar. It follows that if no further mass acretes onto the protostar, $f=1$; and if all the infalling mass interior to the ionization front at $t=t_{3}$ accretes onto the protostar, then $f=2$. The empirical value $f=1.7$ suggests 

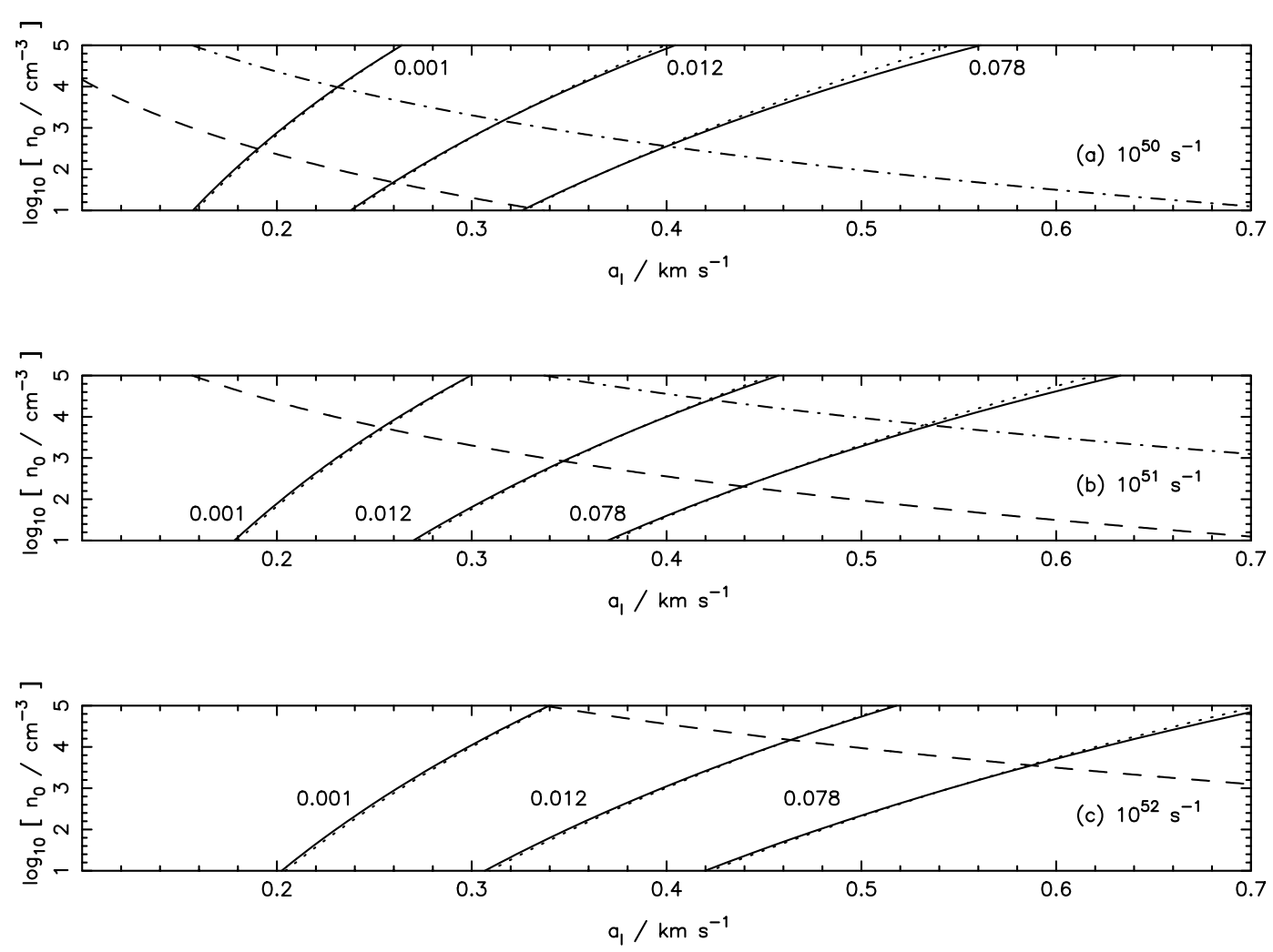

Fig. 1. For a) $\dot{\mathcal{N}}_{\text {Lyc }}=10^{50} \mathrm{~s}^{-1}$; b) $\dot{\mathcal{N}}_{\text {Lyc }}=10^{51} \mathrm{~s}^{-1}$; and c) $\dot{\mathcal{N}}_{\text {Lyc }}=10^{52} \mathrm{~s}^{-1}$, the solid lines show the loci on the $\left(a_{\mathrm{I}}, \log _{10}\left[n_{0}\right]\right)$-plane where free-floating objects of mass $0.001 M_{\odot}$ (one Jupiter mass), $0.012 M_{\odot}$ (the deuterium-burning limit) and $0.078 M_{\odot}$ (the hydrogen-burning limit) form due to photo-erosion of pre-existing cores. The dotted lines show the predictions of the approximate Eq. (40). The dashed (dash-dot) line is the locus below which the volume of the initial core is less than $0.001(0.01)$ of the volume of the whole HII region.

that most of the infalling mass interior to the ionization front at $t=t_{3}$ is accreted onto the protostar.

Equation (40) is plotted as a dotted line in Fig. 1. We see that it is a very good approximation to the numerical results. Given the approximate nature of the whole analysis, Eq. (40) can be viewed as our best estimate of the final protostellar mass.

\section{Discussion}

This exploratory analysis suggests that it is possible for a relatively massive prestellar core which suddenly finds itself immersed in an HII region to be stripped down to the mass of a brown dwarf, or even a planet, by photo-erosion, before it can collapse to form a star. To improve on this analysis, it is probably necessary to resort to numerical modelling. One could then, in principle, (i) use a more realistic configuration for the pre-existing prestellar core; (ii) include the development of the HII region as it overruns the core; (iii) treat properly the early phase when the ionization front advances very rapidly into the outer layers of the core, and the compression wave which is driven into the core; (iv) attempt to capture effects due to departures from spherical symmetry, in particular the radiative transfer aspects; (v) include self-gravity explicitly in the equations of motion. We suspect that the photo-erosion mechanism may be somewhat more effective than our analysis suggests, because firstly, with a prestellar core which is not as centrally condensed as a singular isothermal sphere (e.g. Ward-Thompson et al. 1999; Bacmann et al. 2000), the protostar will not form so quickly, giving the ionization front more time to erode; and secondly, the ionization front will penetrate at higher speed into the less dense central regions.

We can express the final protostellar mass $M_{3}$ in terms of the initial core mass $M_{0}$, by combining Eqs. (40), (16) and (1) to obtain

$M_{3} \simeq \frac{2 f \alpha_{*} G M_{0}^{2}}{3 \alpha_{1} a_{\mathrm{II}}^{2} R_{\mathrm{HII}}}$.

There are two important deductions which we can make from this equation.

First, the ratio of the final protostellar mass to the initial core mass is given by

$\frac{M_{3}}{M_{0}} \sim 10^{-4}\left(\frac{M_{0}}{M_{\odot}}\right)\left(\frac{R_{\mathrm{HII}}}{\mathrm{pc}}\right)^{-1}$.

The photo-erosion process is therefore very effective, in the sense that it strips off most of the mass of the initial core. This makes it a rather wasteful way of creating low-mass protostars, in the sense that it requires a relatively massive initial core to create a single final low-mass protostellar object. In addition, we infer that any intermediate-mass protostars which have formed in the vicinity of a group of OB stars must already have been well on the way to formation before the OB stars switched on their ionizing radiation; otherwise these protostars would have been stripped down to extremely low mass. 
Second, if the number of cores in the mass interval $\left(M_{0}, M_{0}+\mathrm{d} M_{0}\right)$ (i.e. the core mass function) has the form

$\mathcal{N}_{M_{0}} \mathrm{~d} M_{0} \propto M_{0}^{-\gamma} \mathrm{d} M_{0}$,

then the initial mass function for brown dwarves and planetarymass objects formed by photo-erosion has the form

$\mathcal{N}_{M_{3}} \mathrm{~d} M_{3} \propto M_{3}^{-(\gamma+1) / 2} \mathrm{~d} M_{3}$.

Motte et al. (1998) find $\gamma \simeq 1.5$ for $M_{0}<0.5 M_{\odot}$ and $\gamma \simeq 2.5$ for $M_{0}>0.5 M_{\odot}$ for the cores in $\rho$ Ophiuchus, and similar results are reported for the cores in Ophiuchus by Johnstone et al. (2000), for the cores in Serpens by Testi \& Sargent (1998), and for the cores in the Orion B molecular cloud by Motte et al. (2001) and Johnstone et al. (2001). Hence the exponent in the initial mass function for brown dwarves and planetary-mass objetcs formed by photo-erosion should lie in the range -1.25 to -1.75 , with the smaller value $(-1.75)$ obtaining for highermass cores. As the HII region expands and engulfs additional cores, $R_{\mathrm{HII}}$ increases, and therefore for fixed $M_{3}$ the progenitor cores have larger masses $M_{0}$. Consequently, as one moves away from the stars exciting the HII region, the density of brown dwarves and planetary-mass objects formed by photo-erosion should decrease, and their initial mass function should become slightly steeper.

We have omitted from our analysis the possible effect of a stellar wind impinging on a core (A. Burkert, private communication). A simple estimate suggests that a stellar wind would need to be very powerful to have a significant effect. Suppose that the central OB star blows a wind with mass-loss rate $\dot{M}_{\text {wind }} \sim 10^{-4} M_{\odot}$ year $^{-1}$, and speed $v_{\text {wind }} \sim 1000 \mathrm{~km} \mathrm{~s}^{-1}$. The resultant ram pressure acting on a core at radius $R$ is

$P_{\text {wind }}=\frac{\dot{M}_{\text {wind }} v_{\text {wind }}}{4 \pi R^{2}}$.

We can compare this with the ram pressure of the ionized gas flowing off the surface of a core which is exposed to the direct flux of ionizing radiation:

$P_{\mathrm{IF}}=\frac{\dot{\mathcal{N}}_{\mathrm{Lyc}} m a_{\mathrm{II}}}{4 \pi R^{2}}$.

Substituting typical values, we obtain

$$
\begin{aligned}
\frac{P_{\text {wind }}}{P_{\mathrm{IF}}}= & \frac{\dot{M}_{\text {wind }} v_{\text {wind }}}{\dot{\mathcal{N}}_{\mathrm{Lyc}} m a_{\mathrm{II}}}=6 \times 10^{-3}\left(\frac{\dot{M}_{\text {wind }}}{10^{-4} M_{\odot} \text { year }^{-1}}\right) \\
& \times\left(\frac{v_{\text {wind }}}{1000 \mathrm{~km} \mathrm{~s}^{-1}}\right)\left(\frac{\dot{\mathcal{N}}_{\mathrm{Lyc}}}{5 \times 10^{49} \mathrm{~s}^{-1}}\right)^{-1} .
\end{aligned}
$$

This implies that the wind can have little effect. In reality, most of the ionizing flux from the central star is spent maintaining ionization against recombination in the flow of ionized gas from the core, rather than producing new ionizations. Therefore Eq. (46) significantly overestimates the ram pressure of the ionized gas flowing off the core, but probably not by two orders of magnitude. The effect of a stellar wind will be to modify the rate at which the ionized gas flowing off the core can disperse (e.g. Richling \& Yorke 1998; Hollenbach et al. 2000). A bow shock will form on the side of the core facing into the wind (the windward side), and the ionized gas flowing off this side of the core will be swept back in the direction of the wind. This will have the effect of increasing the density, and hence the recombination rate, in the ionized gas flowing off the core, thereby reducing the rate of erosion somewhat. On the leeward side of the core the effect of the wind will be minimal.

\section{Conclusions}

The analysis we have presented in this paper is based on several assumptions and approximations, which, whilst reasonable, are idealizations of what is likely to occur in nature. With this proviso, we infer that, in the central regions of large clusters, free-floating brown dwarves and planetary-mass objects may form when more massive pre-existing cores are overrun by HII regions excited by newly-formed massive stars. The sudden ionization of the surroundings of a core drives a compression wave into the core, creating at its centre a protostar which then grows by accretion. At the same time an ionization front starts eating into the core, thereby removing its outer layers. The final mass of the protostar is determined by a competition between the rate at which it can accrete the infalling envelope, and the rate at which the ionization front can erode the envelope. The simple analysis presented here suggests that in large, dense clusters this will result in the formation of free-floating brown dwarves and/or planetary-mass objects. The process is both robust, in the sense that it operates over a wide range of conditions, and also very effective, in the sense that it strips off most of the mass of the initial core. This means that it is a wasteful way of creating low-mass protostars, because a relatively massive initial core is required to produce a single final brown dwarf or planetary-mass object. It also means that any intermediate-mass protostars which have formed in the vicinity of a group of OB stars must already have been well on the way to formation before the OB stars switched on their ionizing radiation; otherwise these protostars would have been stripped down to extremely low mass.

Note added in proof. The idea that in the vicinity of OB stars final stellar masses may be determinated by photo-erosion (in the manner we have analysed here), and the idea that this is a mechanism for forming free-floating brown dwarves, are originally due to Hester (Hester et al. 1996; Hester 1997).

Acknowledgements. We thank the referee, Doug Johnstone, who drew our attention to a fundamental error in the original version of this paper, and made several other important suggestions which we have incorporated in the final version. We gratefully acknowledge the support of a European Commission Research Training Network, awarded under the Fifth Framework (Ref. HPRN-CT-2000-00155).

\section{References}

Bacmann, A., André, P., Puget, J.-L., et al. 2000, A\&A, 361, 555

Bate, M. R., Bonnell, I. A., \& Bromm, V. 2002, MNRAS, 332, L65

Béjar, V. J. S., Martín, E. L., Zapatero Osorio, M. R., et al. 2001, ApJ, 556, 830

Bertoldi, F. 1989, ApJ, 346, 735 
Bertoldi, F., \& McKee, C. F. 1990, ApJ, 354, 529

Delgado-Donate, E., Clarke, C. J., \& Bate, M. R. 2003, MNRAS, 342, 926

Dyson, J. E. 1968, Ap\&SS, 1, 388

Goodwin, S. P., Whitworth, A. P., \& Ward-Thompson, D. 2004a, A\&A, 414, 633

Goodwin, S. P., Whitworth, A. P., \& Ward-Thompson, D. 2004b, A\&A, 423, 169

Hayashi, C., \& Nakano, T. 1963, Prog. Theor. Phys., 30, 460

Hester J. J. 1997, in Star Formation Near and Far, ed. S. S. Holt \& L. G. Mundy, AIP Conf. Proc., 393, 143

Hester, J. J., Scowen, P. A., Sankrit, R., et al. 1996, AJ, 111, 2349

Hollenbach, D. J., Yorke, H. W., \& Johnstone, D. 2000, in Protostars and Planets, IV (Tucson: University of Arizona Press), ed. V. Mannings, A. P. Boss, \& S. S. Russell, 401

Johnstone, D., Wilson, C. D., Moriarty-Schieven, G., et al. 2000, ApJ, 545,327

Johnstone, D., Fich, M., Mitchell, G. F., \& Moriarty-Schieven, G. 2001, ApJ, 559, 307

Kahn, F. D. 1954, Bull. Astron. Inst. Netherlands, 12, 187

Kahn, F. D. 1969, Physica, 41, 172

Kessel-Deynet, O., \& Burkert, A. 2002, MNRAS, 338, 545

Kroupa, P. 2002, Science, 295, 82

Kumar, S. S. 1963a, ApJ, 137, 1121

Kumar, S. S. 1963b, ApJ, 137, 1126

Lefloch, B., \& Lazareff, B. 1994, A\&A, 289, 559

Lucas, P. W., \& Roche, P. F. 2000, MNRAS, 314, 858

Luhman, K. L., Rieke, G. H., Lada, C. J., \& Lada, E. A. 1998, ApJ, 508,347

Luhman, K. L., \& Rieke, G. H. 1999, ApJ, 525, 440

Luhman, K. L., Rieke, G. H., Young, E. T., et al. 2000, ApJ, 540, 1016
McCaughrean, M. J., Zinnecker, H., Rayner, J. T., \& Stauffer, J. 1995, in The bottom of the Main Sequence - and beyond, ESO Astrophysics Symposium, ed. C. G. Tinney (Springer Verlag), 209

McCaughrean, M. J., Zinnecker, H., Andersen, M., Meeus, G., \& Lodieu, N. 2002, The Messenger, 109, 28

Martín, E. L., Brandner, W., Bouvier, J., et al. 2000, ApJ, 543, 299

Martín, E. L., Zapatero Osorio, M. R., Barrado y Navascués, D., Béjar, V. J. S., \& Rebolo, R. 2001, ApJ, 558, L117

Motte, F., André, P., \& Neri, R. 1998, A\&A, 336, 150

Motte, F., André, P., Ward-Thompson, D., \& Bontemps, S. 2001, A\&A, 372, L41

Nakajima, T., Oppenheimer, B. R., Kulkarni, S. R., et al. 1995, Nature, 378,463

Oort, J. H., \& Spitzer, L. 1955, ApJ, 121, 6

Oppenheimer, B. R., Kulkarni, S. R., Matthews, K., \& Nakajima, T. 1995, Science, 270, 1478

Rebolo, R., Zapatero Osorio, M. R., \& Martín, E. L. 1995, Nature, 377, 129

Reipurth, B., \& Clarke, C. J. 2001, AJ, 122, 432

Richling, S., \& Yorke, H. W. 1998, A\&A, 340, 508

Sandford, M. T. II, Whitaker, R. W., \& Klein, R. I. 1982, ApJ, 260, 183

Shu, F. H. 1977, ApJ, 214, 488

Testi, L., \& Sargent, A. I. 1998, ApJ, 508, L91

Ward-Thompson, D., Motte, F., \& André, P. 1999, MNRAS, 305, 143

Whitworth, A. P., \& Summers, D. 1985, MNRAS, 214, 1

Wilking, B. A., Greene, T. P., \& Meyer, M. R. 1999, AJ, 117, 469

Wilking, B. A., Mikhail, A., Carlson, G., Meyer, M. R., \& Greene, T. P. 2002, AJ, 127, 1131

Zapatero Osorio, M. R., Béjar, V. J. S., Martín, E. L., et al. 2000, Science, 290, 103 